
Jae-Myung Lee*

\title{
Material characteristics of random glass-mat-reinforced thermoplastic under cryogenic thermal cycles
}

https://doi.org/10.1515/secm-2019-0013

Received June 15, 2017; accepted April 11, 2019

Abstract: In this study, cryogenic (77 K) to ambient (293 K) thermal shocks are induced to investigate the material behavior and failure characteristic of a random glass-matreinforced thermoplastic (GMT). The GMT has numerous advantages such as robust thermal conductivity, good mechanical strength, and good impact resistance. Hence, the GMT serves as an insulation material in liquefied natural gas (LNG) carrier-cargo containment systems (CCSs). In this study, 50, 100, 200, 400, and 800 cryogenic thermal cyclic shocks ( $77 \mathrm{~K}$ to $293 \mathrm{~K}$ ) were applied to the fabricated GMT samples. The time for each cycle was $40 \mathrm{~min}$, and it took up to approximately four months to completely apply the thermal cyclic shock to the specimens. The elongation, tensile strength, and elastic modulus of the testing samples obtained from the stress-strain relationship and morphologies were investigated in terms of the number of thermal cyclic shocks and strain rate. Finally, explicit formulae were proposed considering the parameters such as material properties and number of cryogenic thermal cycles to predict the material capabilities under arbitrary loading rates and cryogenic thermal cycles. It was confirmed that the degradation and defects increased with an increase in the number of cryogenic thermal cycles.

Keywords: glass-mat-reinforced thermoplastic; cryogenic; thermal cyclic shock; stiffness degradation

\footnotetext{
Kwang-Jun Park, Haeng-Sung Heo: Gas Technology R\&D Group, Daewoo Shipbuilding \& Marine Engineering, Seoul, Korea Jeong-Hyeon Kim, Seul-Kee Kim, Jin-Ho Bae: Department of Naval Architecture and Ocean Engineering, Pusan National University, Busan, Korea

*Corresponding Author: Jae-Myung Lee: Department of Naval Architecture and Ocean Engineering, Pusan National University, Busan, Korea, E-mail: jaemlee@pusan.ac.kr
}

\section{Introduction}

Cargo-containment systems (CCSs) for liquefied natural gas (LNG) carriers have received significant attention with the increase in demand for LNG, as it is a safe system for transportation across the ocean. In general, a CCS used in LNG carriers, which helps in safely transporting LNG with a temperature of $110 \mathrm{~K}$, can be classified into Moss and membrane-type depending on the component insulating materials and structural layout. Fig. 1(a) shows the most widely employed LNG CCS in ship and offshore industries, which is the Mark-III-type LNG CCS. As shown in the figure, the LNG CCS comprises a metallic-based stainless steel membrane, a composite-based plywood, a reinforced polyurethane foam (RPUF), and laminated adhesives, which help in sustaining the cryogenic temperatures of LNG owing to their robust thermal characteristics. In the CCS, such insulating materials are continuously exposed to repeated wave-induced thermal variations during the loading (110 K) and unloading (293 K) of LNG [1]. In other words, large thermal loads are generated in the component materials of the LNG CCS, causing significant shrinkage and expansion phenomenon in their lifetime. In the past decades, shipbuilding industries have frequently reported sloshing-impact-based cracks, damage, and failure in the conventional LNG CCS. To supplement the mechanical strength of the insulating materials, reinforced insulating materials such as glass-fiber-reinforced polyurethane foam (RPUF) have been widely employed. However, the insulating performance of the RPUF decreases because of the relatively high thermal conductivity of the filler materials, thereby increasing the amount of boil-off rate (BOR). In an attempt to improve the thermal characteristics, an alternative LNG CCS is currently being developed by employing a random glass-mat-reinforced thermoplastic (GMT) as an insulation box, as shown in Fig. 1(b).

The GMT is a polypropylene-based material reinforced with approximately 20 to $50 \%$ of glass mat, which comprises chopped or continuous fibers [2]. The GMT is widely used in automotive applications because of its robust thermal conductivity, good mechanical strength, chemical sta- 
bility, good impact resistance, low cost, and fast processing times [3]. In addition to the automotive industries, the GMT is being developed to employ it in LNG CCS as an alternative insulating material owing to the aforementioned advantages. The conventional insulating materials such as polyurethane foam, plywood, and glass fiber have been extensively studied in terms of their mechanical and thermal properties to employ them in cryogenic (110 K) temperature-based LNG CCS [4-6]. However, the GMT composite, which is rather new, has not been studied experimentally for the application in the LNG CCS as an insulating material.

In the past decades, several studies have been conducted on the material characteristics of the GMT composite. Jung et al. (2017) performed an interlaminar fracturetoughness test to investigate the strain-energy release rates of GMT samples. The test results were verified by comparing the simulation using a finite element analysis with the developed damage model [7]. Kim et al. (2015) manufactured a GMT composite to improve the impact performance with respect to the conventional structural performance of a GMT-based automotive bumper beam [8]. Li et al. (2004) analyzed the high-strain-rate mechanical property of the GMT material using the split Hopkinson pressure-bar technique to investigate the applicability to automobile bodies. The results showed that the GMT material has a potential to replace existing metallic materials [9]. Belingardi et al. (2016) investigated the impact response of a GMT from experimental and simulation viewpoints. In the experiments, the force-displacement and energy-displacement diagrams were analyzed considering the peak load and absorbed energy [10]. Song et al. (2016) studied the effect of the initial fiber alignment on the mechanical properties of GMT composite materials by performing uniaxial tensile test and bending test. The fiber-alignment dependent load-displacement and forcedeflection curves of the GMT were quantitatively analyzed at the ambient temperature [11]. Wolfrath et al. (2005) analyzed the compressive behavior of a GMT at ambient temperature [12]. Renz and Szymikowski (2010) performed tensile tests on advanced GMT composites using a laser extensometer on specimens oriented along the longitudinal and transverse fiber directions. The failure strain was in the range of $2.3-2.5 \%$ [13].

Previous studies focused on the tensile, compressive, and impact resistance of a GMT at ambient or above ambient temperatures for automotive structures. However, to the best of our knowledge, the effects of cryogenic temperature and cryogenic thermal cycle on the GMT have not been investigated. In general, cracks are generated in the composite insulation panels because of the local stress concentrations during the operating condition of the LNG CCS [14]. Hence, it is inevitable to investigate the effect of cryogenic thermal cycles on the material characteristics of the GMT for robust design and safe fabrication of the LNG CCS because the insulation box is constantly exposed to the cryogenic thermal cyclic load during its operation.

In this study, the thermal cyclic shock is applied to the GMT specimens. The tensile tests were then conducted on the GMT specimens to estimate the mechanical degradation in the material properties. Furthermore, the material characteristics, such as the relationship between the number of cryogenic thermal cycles and the tensile stress, were specifically investigated. The explicit formulae were proposed to predict the material capacities under arbitrary cryogenic thermal cycles.

\section{Experimental Preparations}

\subsection{Material and Specimen}

The material investigated in the present study is a polypropylene-based continuous glass mat-reinforced thermoplastic (GMT). Fig. 2 shows the schematic of the GMT material along the thickness direction. It is well known that fiber orientation strongly affects the physical properties of fiber-reinforced composite materials. In the present study, a GMT sheet with randomly aligned glass is used. In addition, the glass fiber content of the GMT is $40 \%$ by weight, which is the widely employed GMT type for structural materials. Fig. 3 shows the test specimen of GMT for the tensile test. As shown in the figure, the test specimens were fabricated according to the ASTM D638-10 standard [15].

\subsection{Measurement for Thermal Equilibrium Time}

Generally, most of the materials expand when exposed to a relatively high temperature, whereas they contract when exposed to a relatively low temperature. However, the tendencies of the materials in attaining the thermal equilibrium time at a certain temperature are different depending on the experimental conditions such as material type, material shape, and dimensions. Hence, the present study analyzed the time required for the GMT material to reach the thermal equilibrium under the liquid-nitrogen condition prior to performing the cryogenic thermal cyclic test. Fig. 4 shows the image and graph pertaining to the thermal 


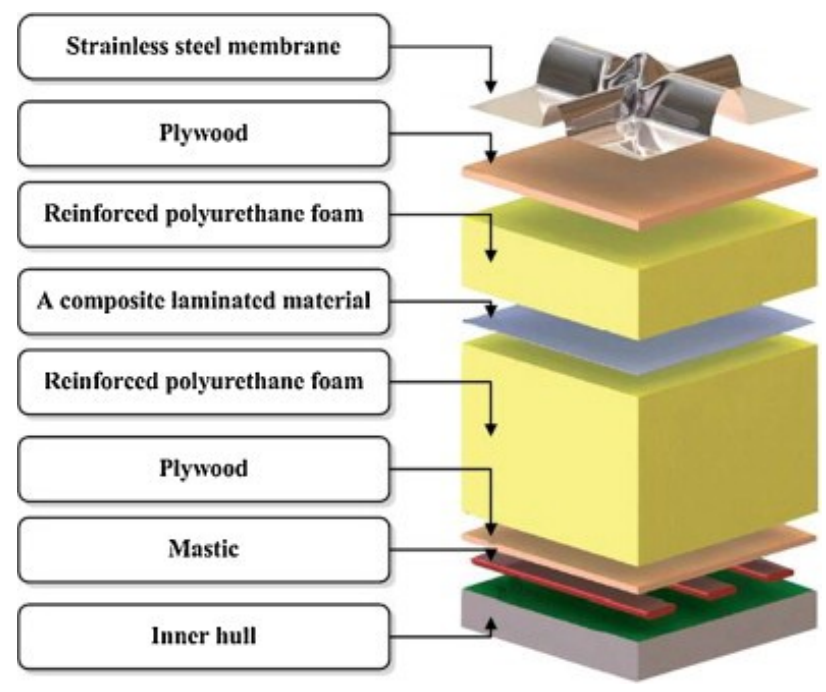

(a)

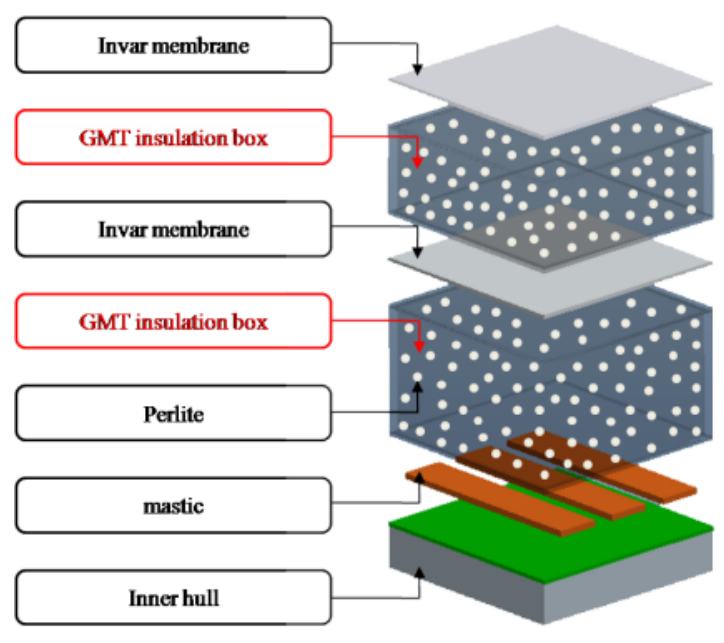

(b)

Figure 1: Schematics of (a) Mark-III-type LNG CCS, and (b) GMTbased LNG CCS [1].

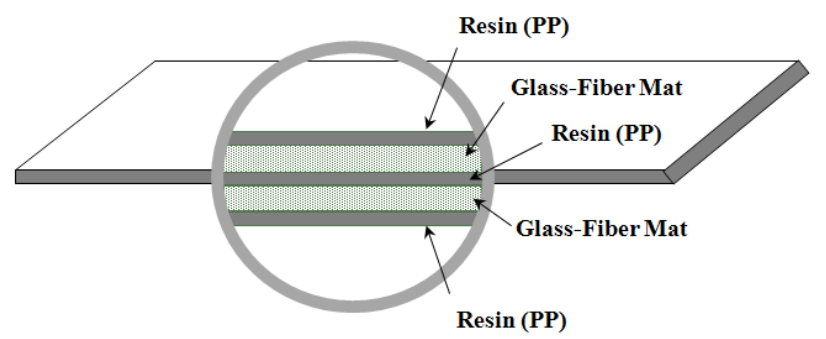

Figure 2: Schematic of tested GMT material.

equilibrium test. The GMT sample placed in liquid nitrogen is fixed to the jigs of the UTM. Thereafter, the variation in the force is recorded as the material shrinks. The graph

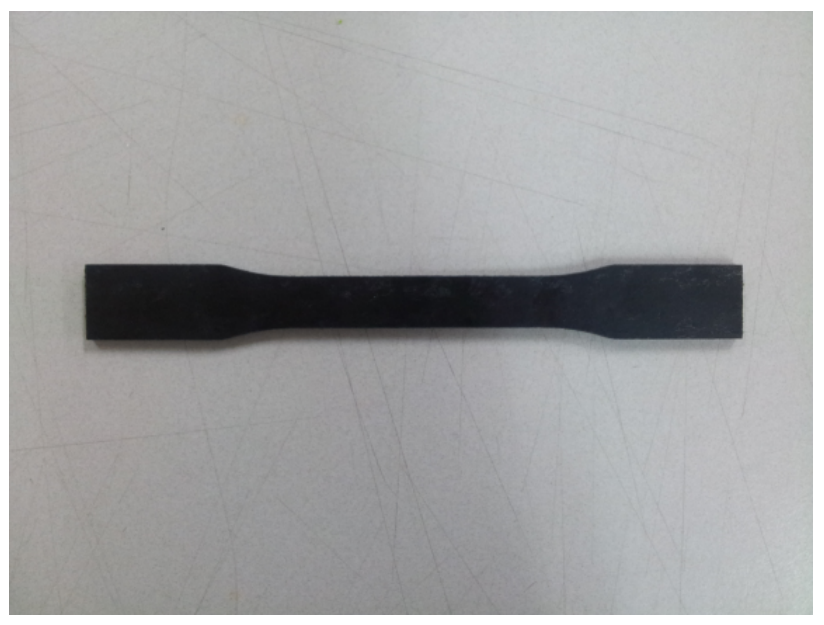

(a)

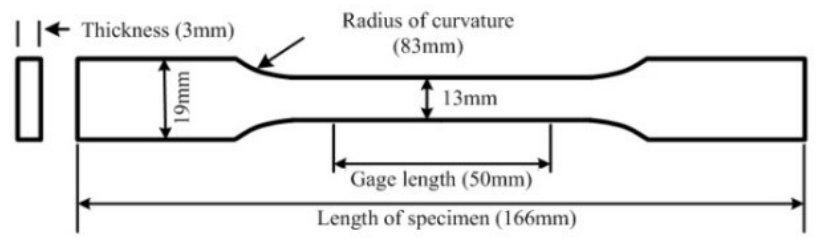

(b)

Figure 3: (a) Image of test sample, and (b) dimensions of test sample

in Fig. 4 shows that the thermal contraction (or thermal equilibrium) is completed after approximately $20 \mathrm{~min}$ into the preliminary test, as the temperature changes from ambient temperature to $77 \mathrm{~K}$. Based on the results, the standard time for the thermal cyclic test was set as $20 \mathrm{~min}$.

\subsection{Thermal Cycle Treatment}

Fig. 5 shows the custom-built LNG immersion cryostat, which was made of austenitic stainless steel (AISI 304) and asbestos insulation materials to sustain the cryogenic temperatures. The liquefied nitrogen was continuously injected into the cryostat to maintain the cryogenic temperature environment $(77 \mathrm{~K})$. The testing samples were immersed into the liquid nitrogen for $20 \mathrm{~min}$, and subsequently, left at room temperature for $20 \mathrm{~min}$. One thermal cycle is equal to $40 \mathrm{~min}$, which is used in preparing for the tensile tests. After the aging process in $\mathrm{LN}_{2}$, the test specimens were exposed to $293 \mathrm{~K}$ for approximately $170 \mathrm{~h}$ (7 days) to sufficiently evaporate the $\mathrm{LN}_{2}$ inside the test specimens. The time for 1 cycle was $40 \mathrm{~min}$, and it took up to approximately four months to age the specimens completely. 

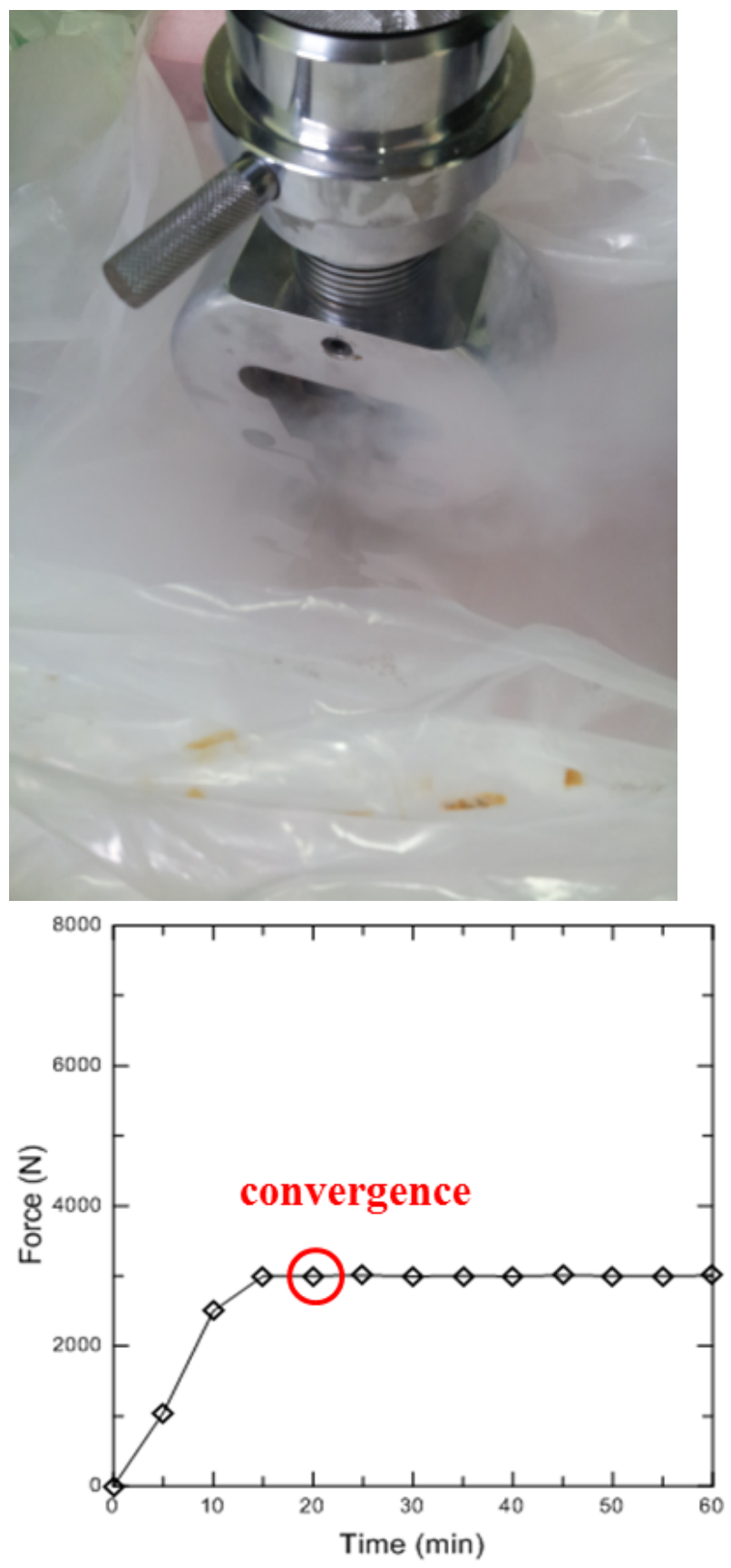

Figure 4: Image and graph for measuring thermal contraction time of the GMT sample in liquid nitrogen.

\subsection{Scenarios for Tensile Test}

Table 1 presents the experimental scenarios. As indicated in the table, the principal parameters for the present investigation were the strain rate and number of cryogenic thermal cycles. The six different thermal cycles $(0,50,100$, $200,400$, and 800$)$ and three different strain rates $\left(10^{-2}\right.$, $10^{-3}$, and $10^{-4} \mathrm{~s}^{-1}$ ) were considered. To obtain a reliable testing result, the experiments were repeated eight times.

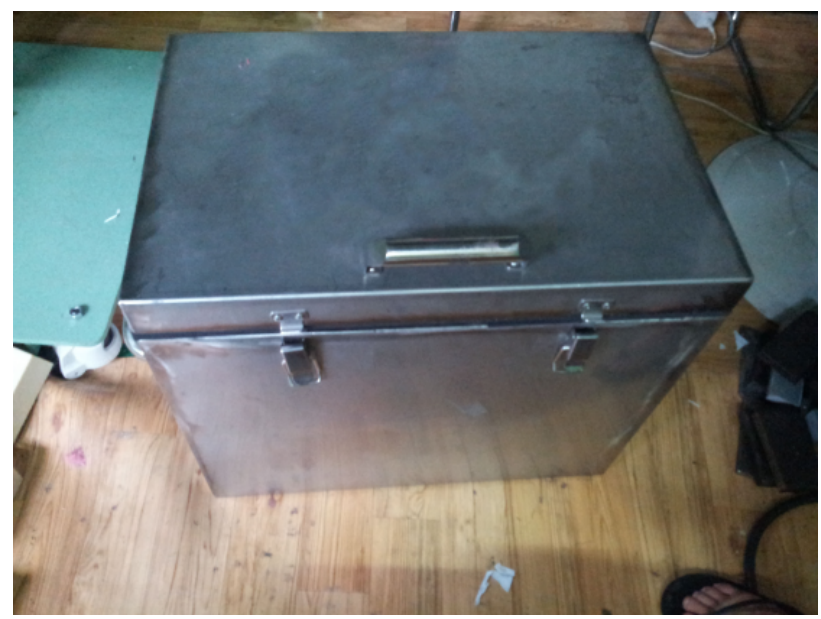

(a)

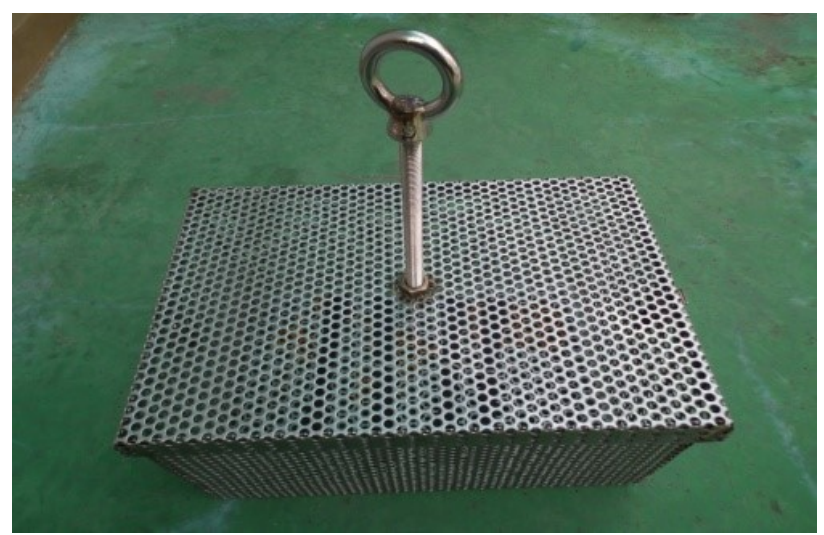

(b)

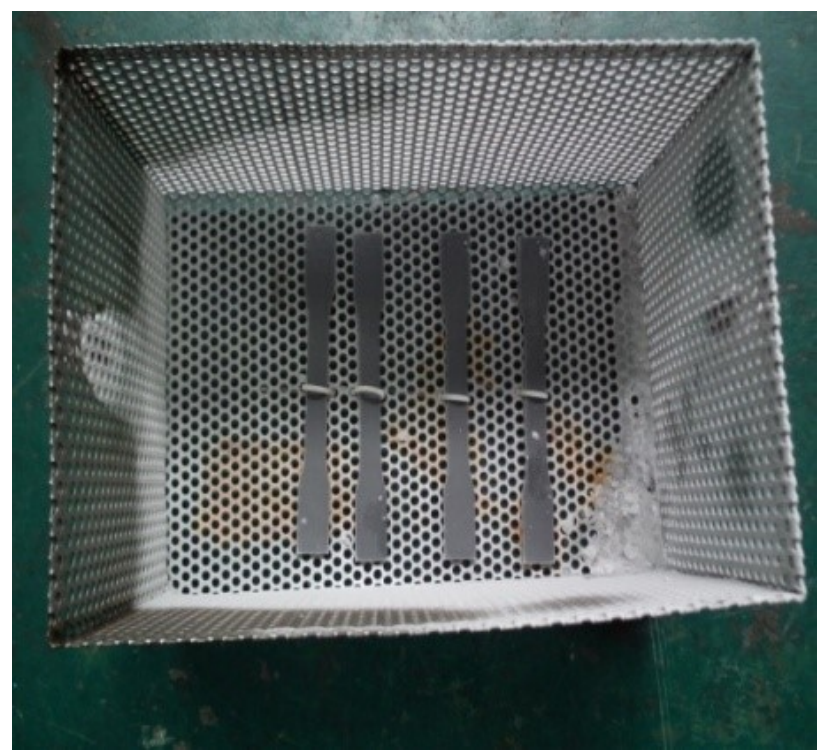

(c)

Figure 5: Images of custom-built (a) cryostat, (b) aging equipment, and (c) aged test specimens 
After the thermal cycle process, the tests were conducted at room temperature.

\section{Results and discussion}

\subsection{Tensile properties}

Table 2 shows the tensile test results of GMT with respect to different strain rates and cryogenic thermal cyclic shock. The GMT exhibits brittle characteristic, which is one of the disadvantages of composite materials after reaching the maximum stress. Previous studies reported on the brittlefracture characteristic of a GMT, demonstrating the sudden fracture, pullout, and matrix fracture of the fiberglass in the GMT $[16,17]$. Table 2 shows that the fracture strain decreases with an increase in the number of thermal cyclic shocks, regardless of the strain-rate conditions. At a strain rate of $10^{-4} \mathrm{~s}^{-1}$, the fracture strain of the as-received sample was 111.7, and the fracture strain of the sample that underwent 800 thermal cycles was 96.1. In other words, the fracture strain decreased by approximately $14.89 \%$ as the number of thermal cyclic shocks was increased from 0 to 800 . Similarly, the fracture strain decreases by approximately $12.52 \%$ and $9.21 \%$ for strain rates of $10^{-3}$ and $10^{-2} \mathrm{~s}^{-1}$, respectively, as the number of thermal cyclic shocks is increased from 0 to 800 . In terms of the strain rate, the results show that a relatively higher strain rate $\left(10^{-2} \mathrm{~s}^{-1}\right)$ leads to a more significant fracture strain compared to a relatively lower strain rate $\left(10^{-4} \mathrm{~s}^{-1}\right)$. The fracture strain is expected to decrease more significantly with the increase in the number of thermal cyclic shocks under higher loading conditions such as sloshing-impact loads induced in the LNG CCS because of waves.

In terms of the tensile strength and elastic modulus, the tensile strength and stiffness steadily decreased as the number of thermal cyclic shocks increased. In other words, the material performance was degraded because of the repeated thermal loads induced between the ambient $(293 \mathrm{~K})$ to cryogenic $(77 \mathrm{~K})$ temperatures. The tensile strength and stiffness reduce because of the microstructural defects and voids in the material due to repeated thermal cyclic shocks. A similar trend in the thermal cyclic shock depending on the material degradation of the insulating material employed in the LNG CCS, plywood composite, had been reported in a previous study [6]. In particular, the tensile strengths of the samples as-received and exposed to 800 thermal cyclic shocks at a strain rate of $10^{-4} \mathrm{~s}^{-1}$ were 111.74 and $95.10 \mathrm{MPa}$, respectively. The results show that the tensile strength decreased by approxi- mately $14.89 \%$ with the increase in the number of thermal cyclic shocks. Similarly, the tensile strengths decreased by approximately 12.52 and $9.21 \%$ at strain rates of $10^{-3}$ and $10^{-2} \mathrm{~s}^{-1}$, respectively, with the increase in the number of thermal cyclic shocks. In terms of the strain rate, the tensile strength and modulus of elasticity were higher than that of the relatively lower strain-rate conditions. The trends such as the relatively higher strength and elastic modulus with the increase in the strain rate were opposite with respect to those observed in the fracture-strain results.

\subsection{Morphological characterization}

To analyze the influence of liquid nitrogen on the GMT specimen and verify the tensile test, through thickness optical microscope images of the GMT are obtained based on the thermal cycle. Each image represents the resin (polypropylene) section. Ten clear images were chosen among each group of thermal cycles and were analyzed based on the assumption that the effects of cracks and voids were most critical. To focus on the influence of liquid nitrogen on GMT, the failure surface of the specimen is not analyzed because voids were formed and the surface was torn. The analysis is performed using a microscope with a magnification of 100x. Figs. 7 to 8 show the images of voids and cracks with respect to the different thermal cycles. For void and crack counting, the direct counting method was used in reference to the optical microscopy results, because it enables sufficient visual observation. This method may be disadvantageous, as it is timeconsuming. However, it is adequately effective and accurate to be adopted for the analysis of optical microscopy results. The method of void and crack counting is conducted in reference to the previous research outcomes. In terms of the judgement criteria employed in this method, the $0.2 \mu \mathrm{m}$ void and crack that can be identified with the naked eye was used as the standard. As the number of cryogenic thermal cycles increases, the voids and cracks grow. Table 3 lists the measured-area volume fraction of the cracks and voids, maximum crack length, and average pole size. As the number of thermal cycle increases, the area volume fraction of the cracks and voids increases linearly. The effect of the average pole size on the tensile properties was negligible, considering that the relationship between the average pole size and the thermal cycle is unclear. 
Table 1: Scenarios for uniaxial tensile test of GMT

\begin{tabular}{llll}
\hline \multirow{2}{*}{ Case } & Strain rate & Cryogenic thermal cycles & \multirow{2}{*}{ No. of samples } \\
\cline { 2 - 3 } & $\left(\mathrm{s}^{-1}\right)$ & (Cycles) & 8 \\
R1-T0 & & 0 & 8 \\
R1-T50 & 50 & 8 \\
R1-T100 & $10^{-2}$ & 100 & 8 \\
R1-T200 & & 200 & 8 \\
R1-T400 & 400 & 8 \\
R1-T800 & 800 & 8 \\
R2-T0 & 0 & 8 \\
R2-T50 & 50 & 8 \\
R2-T100 & 100 & 8 \\
R2-T200 & 200 & 8 \\
R2-T400 & $10^{-3}$ & 400 & 8 \\
R2-T800 & & 800 & 8 \\
R3-T0 & & 0 & 8 \\
R3-T50 & & 50 & 8 \\
R3-T100 & 100 & 8 \\
R3-T200 & & 200 & 8 \\
R3-T400 & 400 & 8 \\
R3-T800 & & 800 & 144 \\
\hline T0tal number of test specimens & & 8 \\
\hline
\end{tabular}

Table 2: Tensile test results for GMT with respect to different strain rates and thermal cycles.

\begin{tabular}{|c|c|c|c|}
\hline \multirow{2}{*}{ Case } & Tensile strength & Elastic modulus & \multirow[t]{2}{*}{ Fracture strain $(\mathrm{mm} / \mathrm{mm})$} \\
\hline & $(\mathrm{MPa})$ & (GPa) & \\
\hline R1-T0 & 127 & 9.53 & 111 \\
\hline R1-T50 & 125 & 9.40 & 108 \\
\hline R1-T100 & 122 & 8.91 & 106 \\
\hline R1-T200 & 119 & 8.68 & 102 \\
\hline R1-T400 & 117 & 8.15 & 100 \\
\hline R1-T800 & 115 & 7.81 & 95.1 \\
\hline R2-T0 & 120 & 8.66 & 120 \\
\hline R2-T50 & 117 & 8.65 & 118 \\
\hline R2-T100 & 114 & 8.09 & 115 \\
\hline R2-T200 & 112 & 7.70 & 112 \\
\hline R2-T400 & 109 & 7.54 & 109 \\
\hline R2-T800 & 105 & 7.04 & 105 \\
\hline R3-T0 & 112 & 6.94 & 127 \\
\hline R3-T50 & 108 & 6.94 & 124 \\
\hline R3-T100 & 106 & 6.46 & 122 \\
\hline R3-T200 & 102 & 6.30 & 119 \\
\hline R3-T400 & 98.6 & 6.20 & 118 \\
\hline R3-T800 & 95.1 & 5.96 & 115 \\
\hline
\end{tabular}

\subsection{Proposal of explicit formulae: Tensile strength}

To predict the tensile strength of the GMT at an arbitrary strain rate and number of cryogenic thermal cy- 
Table 3: Internal defects with respect to cryogenic thermal cycles

\begin{tabular}{llll}
\hline Thermal cycle & Area volume fraction of crack and void & Max. crack length & Ave. pore size \\
\hline (Cycles) & $(\%)$ & $(\mu \mathrm{m})$ & $(\mu \mathrm{m})$ \\
\hline 100 & 0.434 & 8.677 & 0.985 \\
200 & 0.450 & 11.983 & 1.064 \\
400 & 0.728 & 15.646 & 1.043 \\
800 & 1.473 & 26.531 & 1.008 \\
\hline
\end{tabular}

cles, explicit formulae are proposed using $\log$-linear $S-N$ curves. The tensile strength can be derived as a function of the number of cryogenic thermal cycles and strain rate by modifying the fatigue-life prediction equation as follows [18].

$$
\begin{gathered}
\sigma_{\mathrm{T}}=\mathrm{a}_{1}+\mathrm{a}_{2} \log \mathrm{N}_{\mathrm{f}} \\
\sigma_{\mathrm{T}}=a_{3}+a_{4} \log \dot{\epsilon}
\end{gathered}
$$

where $\sigma_{T}$ is the tensile stress, $N_{f}$ is the number of cryogenic thermal cycles, $\dot{\epsilon}$ is the strain rate, and $a_{i}$ is the material parameter.

Fig. 9 shows the relationships between the number of cryogenic thermal cycles and the tensile stress and the strain rate and the tensile stress. Tables 4 and 5 list the obtained material parameters. The figures show that the slopes of the regression lines are largely parallel. This implies that the material degradation rates due to arbitrary number of cryogenic thermal cycles and strain rate were quasi-equal. Hence, the tensile strength and cryogenic thermal fatigue life can be predicted, if the material parameters (termed the principal material parameters) $a_{i}$ are represented as functions of the arbitrary number of cryogenic thermal cycles and strain rate.

Hence, the principal material parameters are represented using the regression formulae as follows.

$$
\begin{aligned}
& \mathrm{a}_{1}=\alpha_{1}+\alpha_{2} \dot{\epsilon} \\
& \mathrm{a}_{2}=\alpha_{3}+\alpha_{4} \dot{\epsilon} \\
& \mathrm{a}_{3}=\beta_{1}+\beta_{2} \mathrm{~N}_{\mathrm{f}} \\
& \mathrm{a}_{4}=\beta_{3}+\beta_{4} \mathrm{~N}_{\mathrm{f}}
\end{aligned}
$$

where $\alpha_{i}$ and $\beta_{i}$ are the associated material parameters of the principal material parameters, namely $a_{i}$. Fig. 10 shows the regression graph of the associated material parameters. Table 6 lists the obtained associated material parameters. Based on the evaluated principal and associated material parameters, the tensile stress could be predicted under an arbitrary number of cryogenic thermal cycles and strain rate.

\subsection{Proposal of explicit formulae: Elastic modulus}

Using the regression analysis, the elastic modulus can be predicted at arbitrary strain rates and number of cryogenic thermal cycles. To predict the elastic modulus of the GMT at arbitrary strain rates and number of cryogenic thermal cycles, explicit formulae are proposed using the log-linear $\mathrm{S}-\mathrm{N}$ curves. The elastic modulus can be derived as a function of the number of cryogenic thermal cycles and strain rate by modifying the fatigue-life prediction equation as follows [18].

$$
\begin{aligned}
& \mathrm{E}_{\mathrm{N}}=\mathrm{b}_{1}+\mathrm{b}_{2} \log \mathrm{N}_{\mathrm{f}} \\
& \mathrm{E}_{\mathrm{N}}=\mathrm{b}_{3}+\mathrm{b}_{4} \log \dot{\epsilon}
\end{aligned}
$$

where $E_{N}$ is the elastic modulus, $N_{f}$ is the number of cryogenic thermal cycles, $\dot{\epsilon}$ is the strain rate, and $b_{i}$ is the material parameter.

Fig. 11 shows the relationships between the number of cryogenic thermal cycles and elastic modulus and the strain rate and the elastic modulus. Table 7 and Table 8 list the obtained material parameters. The regression analysis of the tensile strength shows a similar trend in the elastic modulus.

The figures show that the slopes of the regression lines are largely parallel. Based on these explicit formulae used to calculate the elastic modulus, the elastic modulus and cryogenic thermal fatigue life can be predicted, if the material parameters (termed the principal material parameters) $b_{i}$ are represented as functions of the arbitrary number of cryogenic thermal cycles and strain rate.

Hence, the principal material parameters are represented using the regression formulae as follows.

$$
\begin{aligned}
& \mathrm{b}_{1}=\chi_{1}+\chi_{2} \dot{\epsilon} \\
& \mathrm{b}_{2}=\chi_{3}+\chi_{4} \dot{\epsilon} \\
& \mathrm{b}_{3}=\delta_{1}+\delta_{2} \mathrm{~N}_{\mathrm{f}}
\end{aligned}
$$


Table 4: Material parameters with respect to strain rate

\begin{tabular}{llll}
\hline Parameter & \multicolumn{3}{l}{ Strain rate $\left(\mathrm{s}^{-1}\right)$} \\
\cline { 2 - 4 } & $10^{-4}$ & $10^{-3}$ & $10^{-2}$ \\
\hline$a_{1}(\mathrm{MPa})$ & 127.022 & 134.418 & 137.621 \\
$a_{2}(\mathrm{MPa} /$ cycles $)$ & -4.730 & -4.343 & -3.420 \\
\hline
\end{tabular}

Table 5: Material parameters with respect to number of cryogenic thermal cycles

\begin{tabular}{lllllll}
\hline \multirow{2}{*}{ Parameter } & \multicolumn{6}{l}{ Cryogenic thermal cycles (Cycles) } \\
\cline { 2 - 7 } & 0 & 50 & 100 & 200 & 400 & 800 \\
\hline$a_{3}(\mathrm{MPa})$ & 142.048 & 141.347 & 138.318 & 135.952 & 135.658 & 135.054 \\
\hline$a_{4}(\mathrm{MPa} \cdot \mathrm{s})$ & 3.255 & 3.588 & 3.517 & 3.611 & 3.994 & 4.335 \\
\hline
\end{tabular}

Table 6: Obtained associated material parameters

\begin{tabular}{lllll}
\hline \multirow{2}{*}{ Parameter } & $\alpha_{1}$ (MPa/Cycles) & $\alpha_{2}$ (MPa·s/Cycles) & $\alpha_{3}(\mathrm{MPa})$ & $\alpha_{4}(\mathrm{MPa} \cdot \mathrm{s})$ \\
\cline { 2 - 5 } & 130.131 & 780.856 & -4.609 & 120.255 \\
\hline \multirow{2}{*}{ Parameter } & $\beta_{1}(\mathrm{MPa} \cdot \mathrm{s})$ & $\beta_{2}(\mathrm{MPa} \cdot \mathrm{s} /$ Cycles $)$ & $\beta_{3}(\mathrm{MPa})$ & $\beta_{4}(\mathrm{MPa} /$ Cycles $)$ \\
\cline { 2 - 5 } & 140.121 & $-7.966 \mathrm{E}-03$ & -3.398 & $1.234 \mathrm{E}-03$ \\
\hline
\end{tabular}

Table 7: Material parameters with respect to strain rate

\begin{tabular}{llll}
\hline & \multicolumn{3}{l}{ Strain rate $\left(\mathrm{s}^{-1}\right)$} \\
\cline { 2 - 4 } & $10^{-4}$ & $10^{-3}$ & $10^{-2}$ \\
\hline$b_{1}(\mathrm{MPa})$ & -317.3 & -543.3 & -569.1 \\
\hline$b_{2}(\mathrm{MPa} /$ Cycles $)$ & 8052.6 & 10682.1 & 11606.5 \\
\hline
\end{tabular}

Table 8: Material parameters with respect to number of cryogenic thermal cycles

\begin{tabular}{lllllll}
\hline \multicolumn{7}{c}{ Cryogenic thermal cycles (Cycles) } \\
\cline { 2 - 7 } & 0 & 50 & 100 & 200 & 400 & 800 \\
\hline$b_{3}(\mathrm{MPa})$ & 12267.2 & 12026.8 & 11498.6 & 11142.7 & 10214.5 & 9707.9 \\
\hline$b_{4}(\mathrm{MPa} \cdot \mathrm{s})$ & 563.1 & 535.1 & 532.8 & 518.9 & 422.2 & 400.9 \\
\hline
\end{tabular}

$$
\mathrm{b}_{4}=\delta_{3}+\delta_{4} \mathrm{~N}_{\mathrm{f}}
$$

where $\chi_{i}$ and $\delta_{i}$ are the associated material parameters of the principal material parameters, namely $b_{i}$. Fig. 12 shows the regression graph of the associated material parameters. Table 9 lists the obtained associated material parameters. Based on the evaluated principal and associated material parameters, the elastic modulus can be predicted under arbitrary number of cryogenic thermal cycles and strain rates.

\section{Conclusions}

In this study, the mechanical behavior and failure characteristics of the GMT samples were investigated under ambient-to-cryogenic temperature cycles. The tensile tests were then performed on the GMT test specimens at ambient temperature to investigate the effect of thermal degradation. In addition, the results of the optical microscopic images were obtained to analyze the influence of the liquid nitrogen on the GMT samples. Furthermore, explicit formulae were proposed to predict the material properties at arbitrary number of cryogenic thermal cycles and strain rates. The primary research objectives of the present study and goals for future studies are discussed below. 
Table 9: Obtained associated material parameters

\begin{tabular}{lllll}
\hline \multirow{2}{*}{ Parameter } & $\chi_{1}(\mathrm{MPa} /$ Cycles $)$ & $\chi_{2}(\mathrm{MPa} \cdot \mathrm{s} /$ Cycles $)$ & $\chi_{3}(\mathrm{MPa})$ & $\chi_{4}(\mathrm{MPa} \cdot \mathrm{s})$ \\
\cline { 2 - 5 } & 130.131 & 780.856 & 4.609 & 120.255 \\
\hline \multirow{2}{*}{ Parameter } & $\delta_{1}(\mathrm{MPa} \cdot \mathrm{s})$ & $\delta_{2}(\mathrm{MPa} \cdot \mathrm{s} /$ Cycles $)$ & $\delta_{3}(\mathrm{MPa})$ & $\delta_{4}(\mathrm{MPa} / \mathrm{Cycles})$ \\
\cline { 2 - 5 } & 140.121 & $7.966 \mathrm{E}-03$ & -3.398 & $1.234 \mathrm{E}-03$ \\
\hline
\end{tabular}

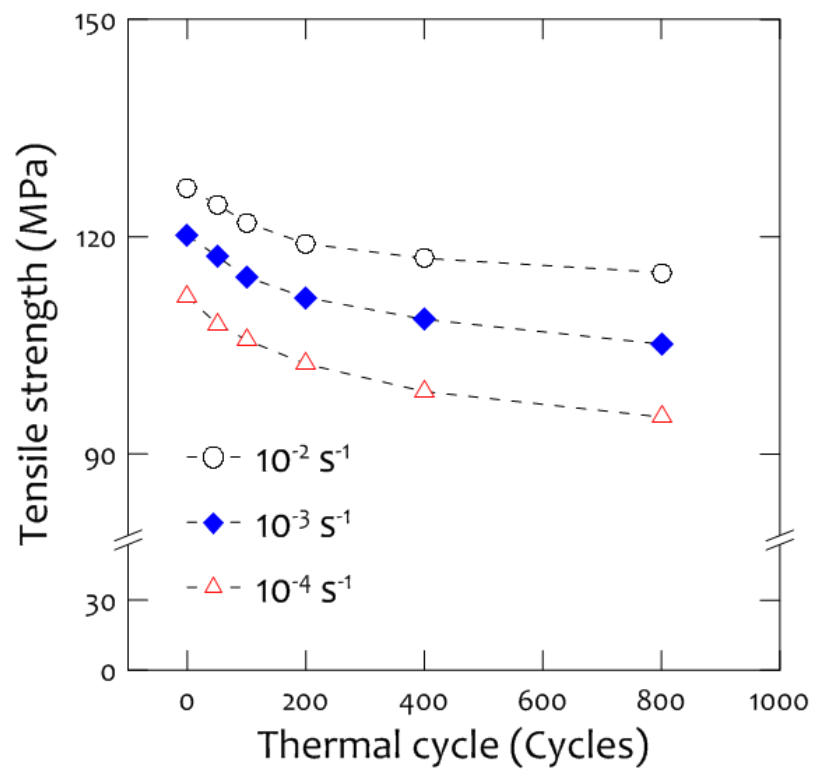

(a)

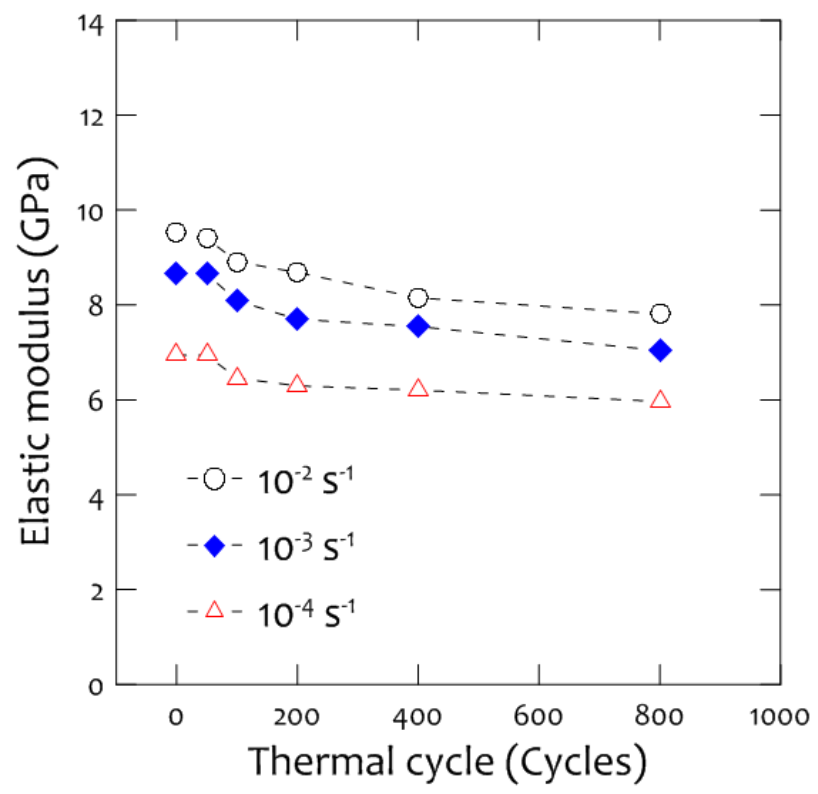

(b)

Figure 6: Degradation of (a) tensile strength, and (b) elastic modulus depending on thermal cycle and elastic modulus.
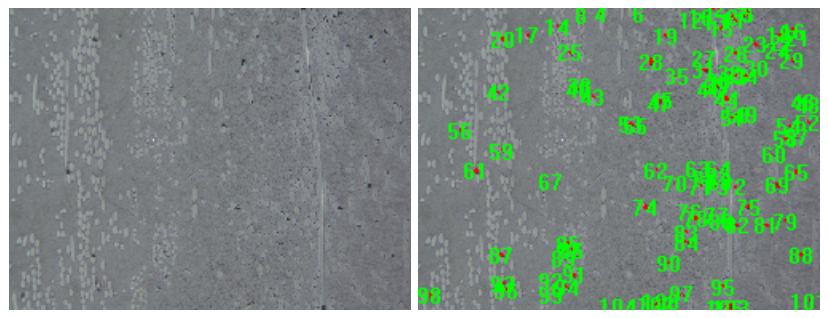

(a)
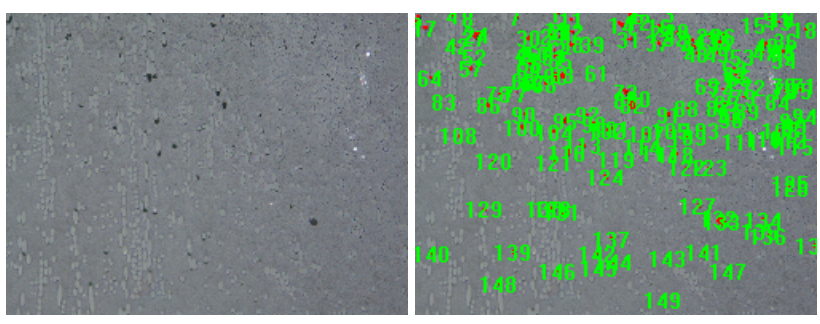

(b)

Figure 7: Representative optical microscope images and measured defects at (a) 100 and (b) 200 cycles
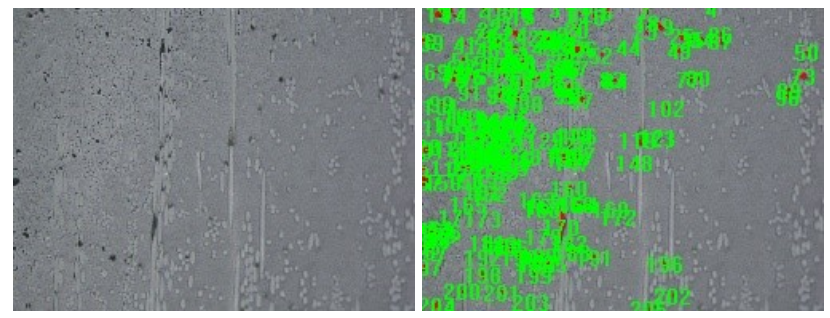

(a)
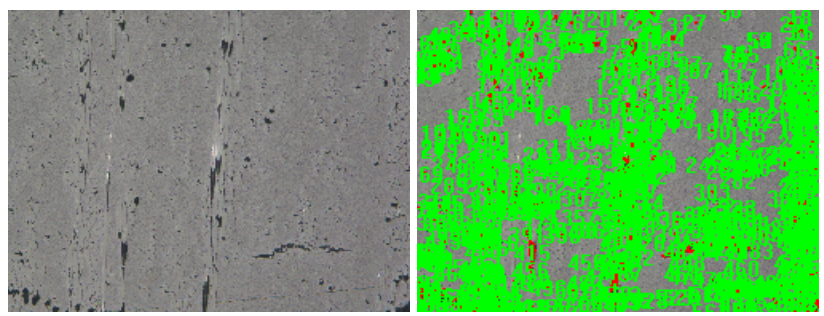

(b)

Figure 8: Representative optical microscope images and measured defects at (a) 400, and (b) 800 cycles 


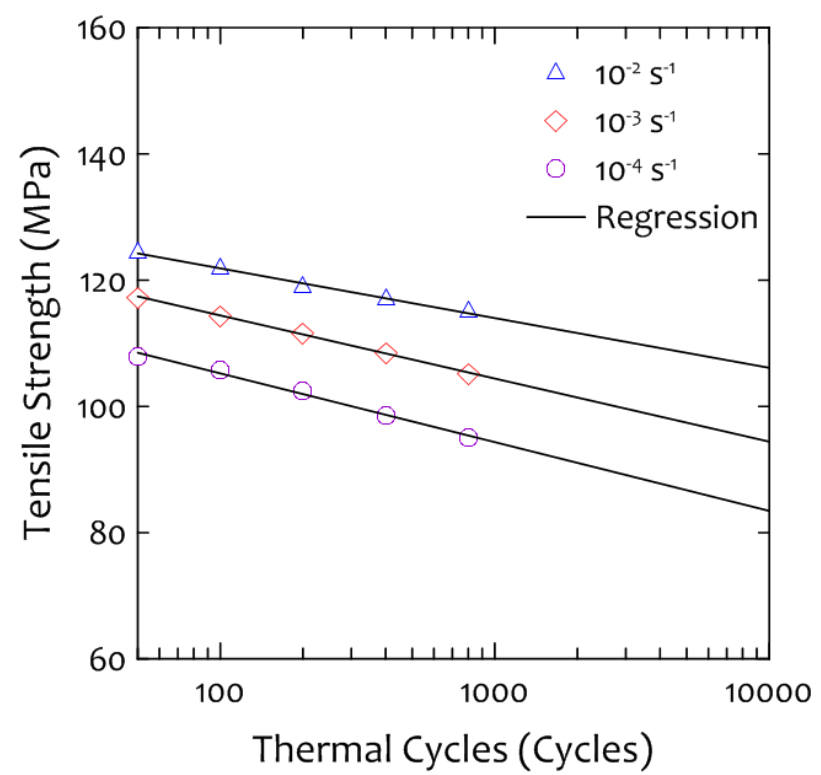

(a)

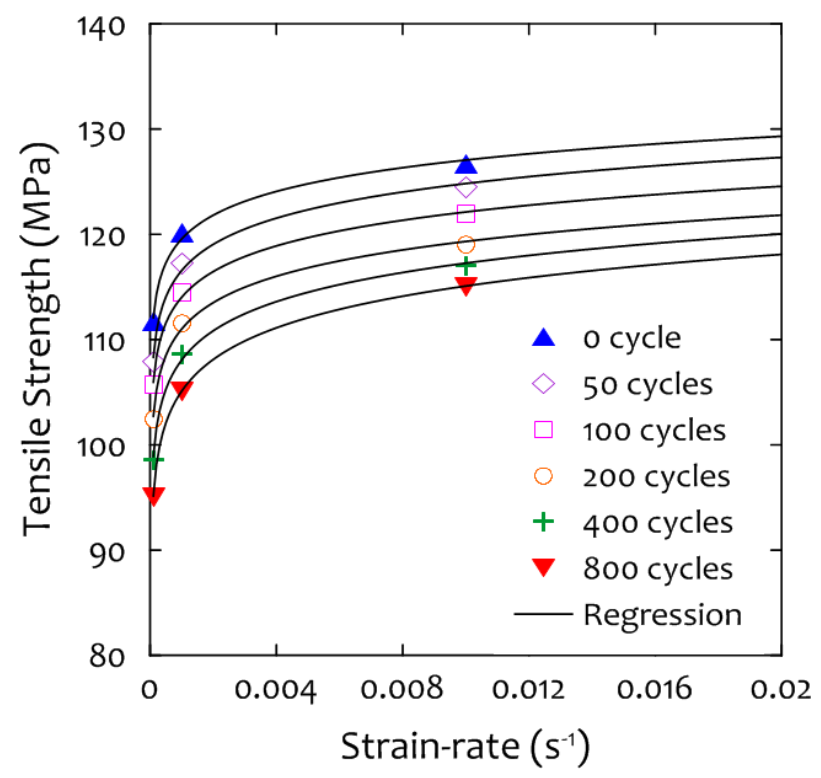

(b)

Figure 9: Relationship of (a) Cryogenic thermal cycles and tensile stress, and (b) strain rate and tensile stress for different strain rates and number of thermal cycles

To predict the tensile strength of the GMT under an arbitrary number of cryogenic thermal cycles and strain rates, empirical formulae, and principal/associated material parameters were proposed.

- The material degradation was clearly observed in the GMT samples with an increase in the number of thermal cyclic shocks. The tensile strength of the GMT decreased by $16 \%$ as the number of cryogenic thermal cycles reached 800 .

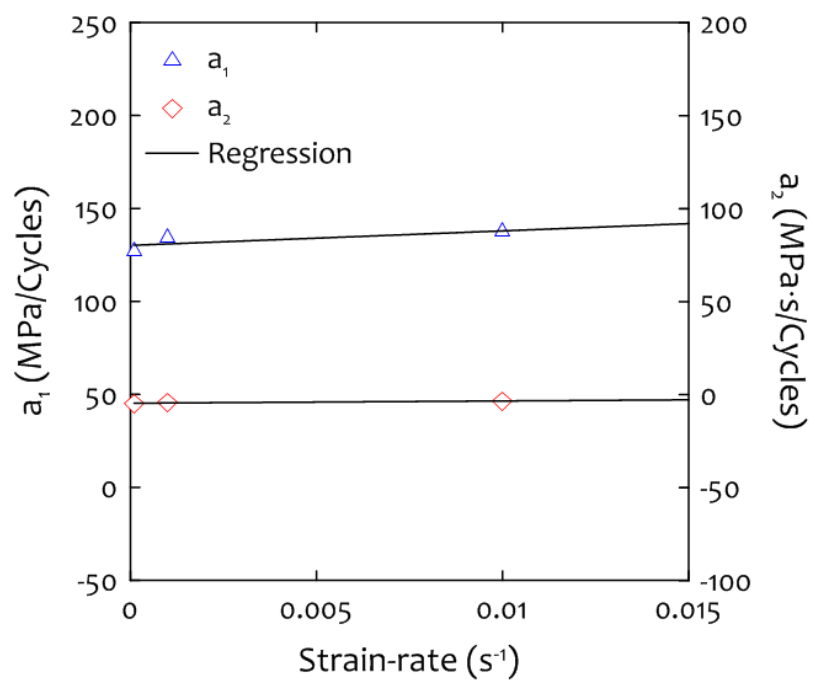

(a)

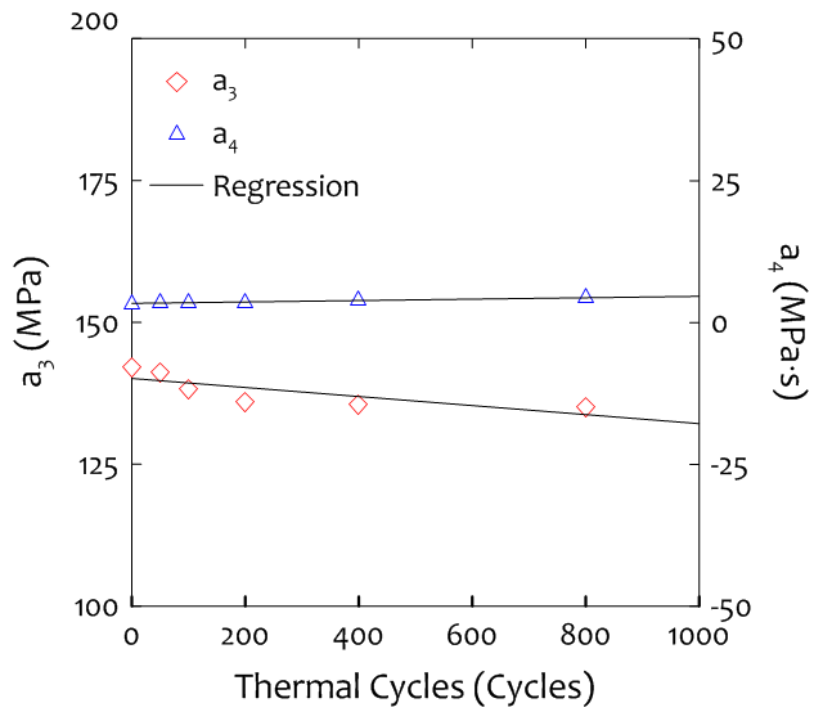

(b)

Figure 10: Regression graph of material parameters

- The tensile strength and elongation increase with an increase in the strain rate. However, the degradation rates were largely the same in all the strain-rate cases.

- The area volume fraction of the cracks and voids increased linearly with the increase in the number of thermal cyclic shocks. The effect of the average pole size on the tensile properties was negligible as the relationship between the average pole size and the thermal cycle was unclear.

- To predict the tensile strength of the GMT under arbitrary number of cryogenic thermal cycles and strain rates, empirical formulae, and principal/associated material parameters were proposed. 


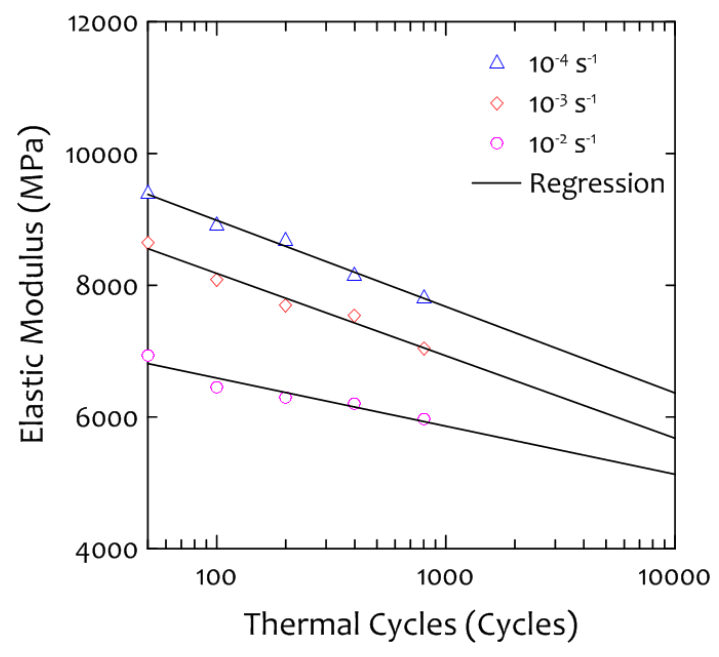

(a)

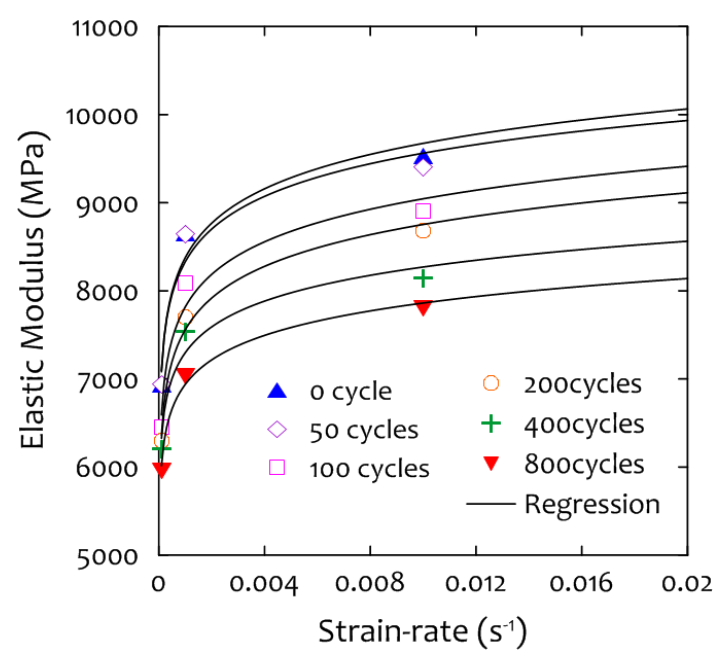

(b)

Figure 11: Relationship between (a) Cryogenic thermal cycles and elastic modulus, and (b) strain rate and elastic modulus for different strain rates and numbers of thermal cycles

The explicit formulae and material constants proposed in this study will be helpful in designing robust and safe GMT-based structures under cryogenic thermal fatigue and loading conditions. In addition, the results are not widely applicable for composite materials, but for preceding research of material behaviors. In future studies, further scenario tests on cryogenic-temperature-aged GMT materials will be conducted to obtain specific material characteristics. In addition, strain-rate-dependent constitutive equations for describing the nonlinear material behavior will be proposed. Furthermore, the developed equations will be implemented in finite element analysis to computationally analyze the nonlinear material behavior.

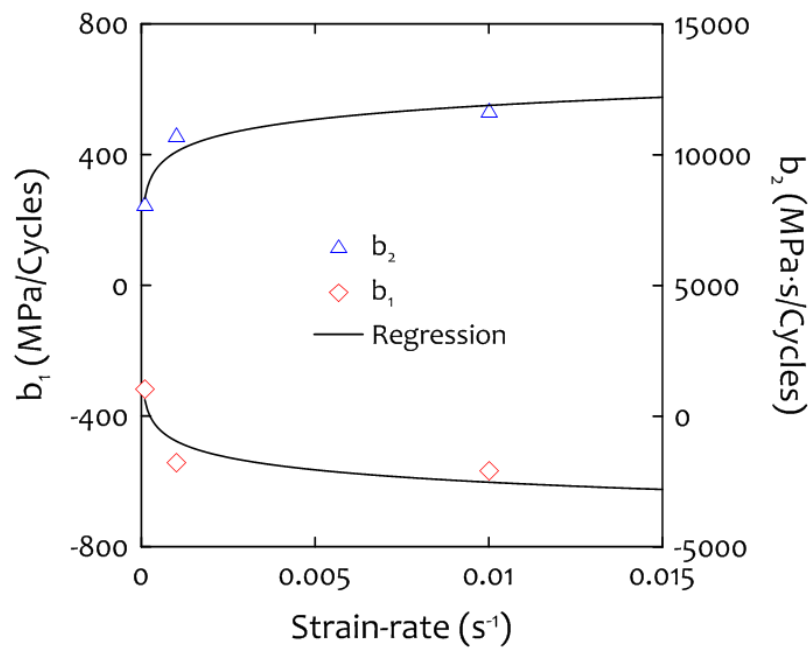

(a)

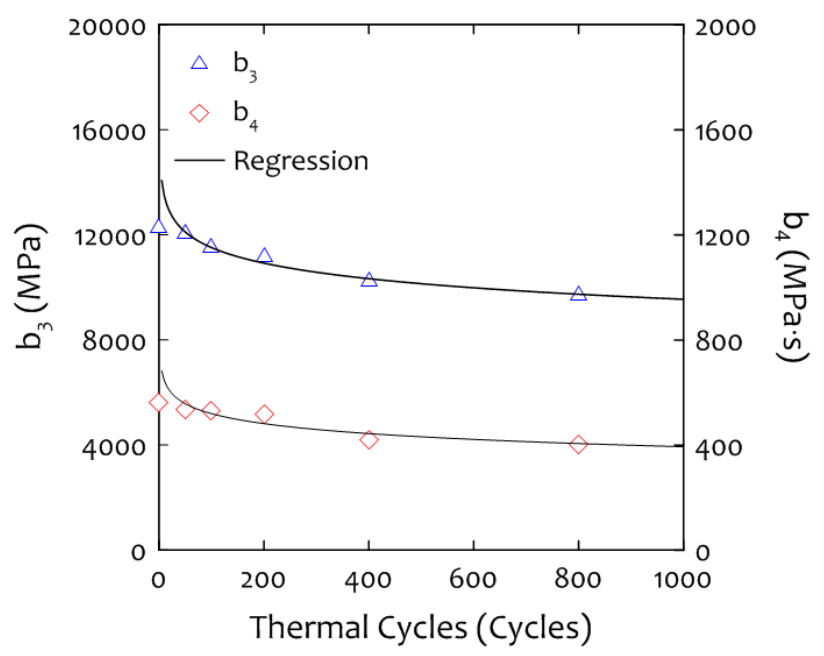

(b)

Figure 12: Regression graph of material parameters

Acknowledgements: This work was supported by the National Research Foundation of Korea (NRF) grant funded by the Ministry of Science and ICT (MSIT) (No. 2018R1A2B6007403). This work was supported by the National Research Foundation of Korea (NRF) grant funded by the Korea government (MSIT) through GCRC-SOP (No. 2011-0030013).

\section{Declaration of Conflicting Interests}

The author(s) declared no potential conflicts of interest with respect to the research, authorship, and/or publication of this article.

\section{Funding}

The author(s) received no financial support for the research, authorship, and/or publication of this article. 


\section{References}

[1] Kim JH, Park WS, Chun MS, et al. Effect of pre-straining on lowtemperature mechanical behavior of AISI 304L. Mater Sci Eng A 2012; 543: 50-57.

[2] Dasappa P, Sullivan PL and Xiao X. Temperature effects on creep behavior of continuous fiber GMT composites. Compos Part $A$ 2009; 40: 1071-1081.

[3] Kim JW and Lee DG. Creep and tensile properties of press molding joint GMT-sheets. T Nonferr Metal Soc 2011; 21: 170-174.

[4] Park SB, Lee CS, Choi SW, et al. Polymeric foams for cryogenic temperature application: temperature range for non-recovery and brittle-fracture of microstructure. Compos Struct 2016; 136: 258-269.

[5] Kim JM, Kim SK, Kim, JM, et al. Numerical model to predict deformation of corrugated austenitic stainless steel sheet under cryogenic temperatures for design of liquefied natural gas insulation system. Mater Des 2014; 57: 26-39.

[6] Kim JH, Park DH, Lee CS, et al. Effects of cryogenic thermal cycle and immersion on the mechanical characteristics of phenolresin bonded plywood. Cryogenics 2015; 72: 90-102.

[7] Jung KH, Kim DH, Kim HJ, et al. Finite element analysis of a low-velocity impact test for glass fiber-reinforced polypropylene composites considering mixed-mode interlaminar fracture toughness. Compos Struct 2017; 160: 446-456.

[8] Kim DH, Kim HG and Kim HS. Design optimization and manufacture of hybrid glass/carbon fiber reinforced composite bumper beam for automobile vehicle. Compos Struct 2015; 131: 742-752.
[9] Li Y, Lin Z, Jiang A, et al. Experimental study of glass-fiber mat thermoplastic material impact properties and lightweight automobile body analysis. Mater Des 2004; 25: 579-585.

[10] Belingardi G, Beyene AT and Jichuan D. Energy absorbing capability of GMT, GMTex and GMT-UD composite panels for static and dynamic loading - Experimental and numerical study. Compos Struct 2016; 143: 371-387.

[11] Song Y, Gandhi U, Koziel A, et al. Effect of the initial fiber alignment on the mechanical properties for GMT composite materials. J Thermoplast Compos (DOI: 10.1177/0892705716681400).

[12] Wolfrath J, Michaud V and Manson JAE. Deconsolidation in glass mat thermoplastics: Influence of the initial fibre/matrix configuration. Compos Sci Technol 2005; 65: 1601-1608.

[13] Renz R and Szymikowski R. Locally resolved hysteresis measurement of advanced glass-mat thermoplastic composites. Int J Fatigue 2010; 32: 174-183.

[14] Melcher RJ and Johnson WS. Mode I fracture toughness of an adhesively bonded composite-composite joint in a cryogenic environment. Compos Sci Technol 2007; 67: 501-506.

[15] ASTM. Standard test method for tensile properties of plastics. D638 - 10 (2010) 1-16.

[16] Lee NJ and Jang JS. The use of a mixed coupling agent system to improve the performance of polypropylene-based composites reinforced with short-glass-fibre mat. Compos Sci Technol 1997; 57: 1559-1569.

[17] Bartus SD and Vaidya UK. Performance of long fiber reinforced thermoplastics subjected to transverse intermediate velocity blunt object impact. Compos Struct 2005; 67: 263-277.

[18] Dowling ND. Mechanical Behavior of Materials. Pearson Prentice Hall. 2007. 